\title{
Trois scénarios de décroissance de la population québécoise
} Caractéristiques et incidences sur la population active

\section{Three Projections of Population Decline for Quebec}

Characteristics and Implications for the Working Population

\section{Tres escenarios de decrecimiento de la población quebequense} Características e incidencias sobre la población activa

\section{Hervé Gauthier}

Volume 15, numéro 2, octobre 1986

La décroissance démographique et ses implications

URI : https://id.erudit.org/iderudit/600594ar

DOI : https://doi.org/10.7202/600594ar

Aller au sommaire du numéro

Éditeur(s)

Association des démographes du Québec

ISSN

0380-1721 (imprimé)

1705-1495 (numérique)

Découvrir la revue

Citer cet article

Gauthier, H. (1986). Trois scénarios de décroissance de la population québécoise : caractéristiques et incidences sur la population active. Cahiers québécois de démographie, 15(2), 181-212. https://doi.org/10.7202/600594ar
Résumé de l'article

Après avoir décrit différents scénarios de décroissance de la population du Québec, l'auteur fournit des exemples de déclin observés au XX ${ }^{\mathrm{e}}$ siècle, et souligne que la possibilité de recourir à l'immigration pour éviter la décroissance pourrait être limitée si la population ne modifie pas son attitude plutôt négative à l'égard d'un accroissement de l'immigration. Il analyse ensuite les implications de la décroissance sous quatre aspects : la taille de la population active, sa composition par sexe et par âge, le taux brut d'activité et le rapport de dépendance économique. 
Cahiers québécois de démographie

Vol. 15, no 2, octobre 1986

\title{
Trois scénarios de décroissance de la population québécoise: caractéristiques et incidences sur la population active
}

\author{
Hervé GAUTHIER*
}

\section{INTRODUCTION}

Il y a maintenant près de 15 ans que l'indice synthétique de fécondité de la population québécoise est sous le seuil de remplacement des générations. Ce qui était perçu au début comme un phénomène temporaire prend de plus en plus un caractère permanent. On sait qu'à long terme un tel niveau de fécondité entraînera un accroissement naturel négatif et une diminution de la population totale, à moins que les migrations ne viennent compenser l'insuffisance de la fécondité. Le présent texte veut explorer diverses facettes de la décroissance démographique, particulièrement en ce qui concerne la population active.

La première section est consacrée à la description du contexte démographique général dans lequel se présente le problème actuel de la décroissance. Pour ce faire, nous commençons par voir ce que pourrait être celle-ci, en examinant les divers scénarios d'évolution démographique qui impliquent une décroissance de la population québécoise. Nous utiliserons à cet effet les projections démographiques du Bureau de la statistique du Québec. D'autres travaux avaient déjà fourni des scénarios à long terme comportant une décroissance de la population (OPDQ, 1980; Gauthier et Malo, 1983), mais les projections du BSQ publiées en 1984 présentent des hypothèses de fécondité plus faible, ce qui correspond davantage au niveau observé au cours des dernières années. Même si la possibilité d'une décroissance éventuelle fait figure de phénomène nouveau dans 1'histoire du Québec, ce n'est pas le cas partout en Amérique du Nord. Des provinces canadiennes, ainsi que des Etats américains, ont connu au cours du XXe siècle des périodes de diminution de la population. Il est intéressant de relever ces cas de décroissance, l'ampleur que le phénomène a eue et sa durée, tout en soulignant cependant que la source du phénomène était bien différente de ce qui s'annonce dans

Bureau de la statistique du Québec, Service de 1'analyse et de la prévision démographiques, Québec.

Les idées exprimées dans ce texte sont celles de 1'auteur et n'engagent pas la responsabilité du BSQ. 
1 'avenir. Devant la fajblesse et éventuellement 1 'insuffisance de 1'accroissement naturel, la première réaction est souvent de proposer de faire appel à une immigration plus abondante. Il est donc normal de se demander quelle est l'attitude de la population à l'égard de l'immigration : nous terminerons cette section sur cet aspect de la question.

Nous nous penchons ensuite sur I'incidence qu'aurait une telle évolution démographique dans le domaine crucial de la population active. Pour ce faire, nous devons examiner comment passer des projections démographiques aux projections de population active : la deuxième section de notre article est consacrée à I'opérationnalisation des hypothèses de prévision de la population active. Après avoir brièvement décrit l'évolution passée des effectifs et de la structure de celle-ci, nous expliciterons nos hypothèses quant à son évolution future.

Les résultats concernant l'évolution de la population active prévue selon chacune de ces hypothèses sont présentés dans la troisième section. Ces résultats seront commentés sous quatre angles : la taille de la population active, sa composition par sexe et par âge, le taux brut d'activité et le taux de dépendance économique.

\section{LA TOILE DE FOND}

\subsection{Le virage démographique}

Les conditions qui mènent à 1 a décroissance démographique sont déjà en place. D'un côté, la fécondité est inférieure au seuil de remplacement des générations. Le plus récent indice du moment disponible, celui de l'année 1984, se situe à 1,45 enfant par femme; par aflleurs, si on estime la fécondité qu'elle pourrait encore avoir, la génération féminine née en 1953-1954 n'aurait que 1,76 enfant environ, ce qui est bien en dessous du minimum requis $(2,1)$. Les informations concernant les générations plus récentes indiquent une poursuite de la baisse. D'un autre côté, malgré une amélioration substantielle de son solde migratoire, le Québec demeure toujours déficitaire dans ses échanges avec l'extérieur; on ne sait pas si cette amélioration est temporaire ou est appelée à durer.

En dépit de ces conditions adverses, la population du Québec continue de crô̂tre. En fait, la décroissance est retardée en raison de la structure par âge favorable à la natalité et défavorable à la mortalité. Pendant un certain temps encore, cette structure par âge va donner un accroissement naturel positif. La durée du sursis dont bénéficie le Québec et qui est 
héritée de la fécondité passée, dépend pour une large part du niveau de fécondité des prochaines années. Les plus récentes perspectives démographiques du Québec permettent d'analyser cette question.

Les hypothèses migratoires à $1 \mathrm{a}$ base de ces perspectives varient de façon significative d'un scénario à l'autre, du moins pour les premières années de la projection. Ainsi, le solde migratoire est établi à -17000 dans le scénario faible en 1984-1985, première année de projection, et à -11000 dans le scénario fort; en 1991-1992, le solde touche respectivement -9000 et 2000 . A long terme cependant, les trois scénarios ne se différencient presque plus (écart de 1600 dans le solde migratoire des scénarios faible et fort).

En ce qui concerne la fécondité, les trois scénarios débutent au même point, soit un niveau correspondant à un indice synthétique de 1,45 enfant par femme en 1983. Dans chaque scénario, l'indice évolue en baisse ou en hausse pour se stabiliser à un niveau constant : à 1,35 et 1,60 en 1986, respectivement pour les scénarios faible et moyen, et à 1,80 en 1990 pour le scénario fort. Dans le présent article, nous utiliserons en outre, à titre de référence, un quatrième scénario caractérisé par une fécondité de 2,10 qui serait atteinte en 1996. Ces niveaux de fécondité sont maintenus jusqu'au terme de la période considérée ici, soit 2061.

Quant à l'hypothèse de mortalité, elle se définit en fonction d'une amélioration non négligeable de l'espérance de vie, qui passerait entre 1981 et 2001 de 71,0 à 73,5 ans pour le sexe masculin, et de 78,7 à 82,2 ans pour le sexe féminin. La même hypothèse est reprise dans chaque scénario, sauf le quatrième scénario basé sur une table de mortalité légèrement différente (espérance de vie à long terme de 73,2 et 82,8 ans, respectivement).

En somme, dans une perspective de très long terme, ces scénarios d'évolution future de la population québécoise servent surtout à mettre en évidence l'influence de la fécondité. Le tableau 1 présente les grandes lignes de ce que serait l'évolution quinquennale de la taille de la population du Québec selon chacun des scénarios. La discussion portera cependant sur l'évolution annuelle, afin de souligner les différences entre scénarios.

On constate sans grande surprise que les trois scénarios (A : "faible", B : "moyen", C : "fort") pour lesquels I'indice synthétique de fécondité est inférieur à 2,1 conduisent tous à une diminution des effectifs de la population québécoise. Bien qu'en vertu des hypothèses posées au départ la décroissance démographique soit inéluctable, ce phénomène est loin de débuter 
Tableau 1

Population du Québec et taux d'accroissement annuel moyen, 1951-1981, et selon quatre scénarios, 1986-2001

\begin{tabular}{|c|c|c|c|c|c|c|c|c|}
\hline \multirow{2}{*}{$\begin{array}{l}\text { Période } \\
1951-1956 \\
1956-1961 \\
1961-1966 \\
1966-1971 \\
1971-1976 \\
1976-1981\end{array}$} & \multicolumn{4}{|c|}{$\begin{array}{l}\text { Population au début } \\
\text { de la période } \\
\text { (en milliers) }\end{array}$} & \multicolumn{4}{|c|}{$\begin{array}{c}\text { Taux d'accroissement } \\
\text { annuel moyen par période } \\
\text { (pour 100) }\end{array}$} \\
\hline & \multicolumn{4}{|c|}{$\begin{array}{l}4056 \\
4628 \\
5259 \\
5781 \\
6028 \\
6234\end{array}$} & \multicolumn{4}{|c|}{$\begin{array}{l}2,7 \\
2,6 \\
1,9 \\
0,8 \\
0,7 \\
0,6\end{array}$} \\
\hline & \multicolumn{4}{|c|}{ Scénario } & \multicolumn{4}{|c|}{ Scénario } \\
\hline & A & B & $\mathrm{C}$ & $\mathrm{D}$ & A & B & $\mathrm{C}$ & D \\
\hline $1981-1986$ & 6438 & 6438 & 6438 & 6438 & 0,5 & 0,5 & 0,5 & 0,5 \\
\hline $1986-1991$ & 6585 & 6611 & 6617 & 6617 & 0,3 & 0,6 & 0,7 & 0,7 \\
\hline $1991-1996$ & 6683 & 6808 & 6860 & 6860 & 0,2 & 0,3 & 0,7 & 0,8 \\
\hline $1996-2001$ & 6737 & 6965 & 7103 & 7142 & 0,0 & 0,3 & 0,5 & 0,7 \\
\hline $2001-2006$ & 6739 & 7065 & 7290 & 7400 & $-0,1$ & 0,2 & 0,4 & 0,6 \\
\hline $2006-2011$ & 6708 & 7127 & 7437 & 7612 & $-0,2$ & 0,1 & 0,3 & 0,4 \\
\hline $2011-2021$ & 6640 & 7158 & 7552 & 7779 & $-0,4$ & $-0,1$ & 0,2 & 0,4 \\
\hline $2021-2031$ & 6386 & 7120 & 7706 & 8057 & $-0,7$ & $-0,3$ & $-0,0$ & 0,2 \\
\hline $2031-2041$ & 5981 & 6918 & 7703 & 8219 & $-0,9$ & $-0,5$ & $-0,2$ & 0,0 \\
\hline $2041-2051$ & 5480 & 6603 & 7574 & 8242 & $-1,0$ & $-0,5$ & $-0,2$ & 0,0 \\
\hline $2051-2061$ & 4975 & 6270 & 7427 & 8268 & $-0,9$ & $-0,5$ & $-0,2$ & 0,1 \\
\hline 2061 & 4547 & 5982 & 7309 & 8344 & & & & \\
\hline
\end{tabular}

Sources : Recensements du Canada et projections du Bureau de la statistique du Québec. 
au même moment dans les trois scénarios et il n'a pas du tout la même ampleur à 1 'horizon 2061. Dans le scénario A, la population atteint un maximum dès 1999 avec 6743000 personnes et 60 années plus tard la population est réduite du tiers. A noter que le début de la décroissance serait retardé de quelques années si le solde migratoire n'était pas négatif, puisque le déficit des naissances sur les décès ne survient qu'en 2003. Dans le scénario $B$, le déclin de la population survient presque en même temps que 1 'accroissement naturel négatif, soit en 2014. La population est alors de 7161000 personnes et elle diminue de $16 \%$ entre cette année-1à et la fin de la période de projection. Dans le scénario $C$, la décroissance ne se produit qu'à partir de 2026 ( 1 'accroissement naturel est négatif depuis quelques années déjà) : la population de 7727000 personnes décroît d'à peine $5 \%$ jusqu'en 2061. Les différences sont donc grandes entre les trois scénarios de décroissance. Seulement 13 années nous séparent de l'éventualité la plus rapprochée, mais 40 de l'éventualité la plus lointaine. D'autre part, dans le scénario le plus faible, la population diminue fortement après avoir atteint un sommet. La diminution est faible dans le cas du scénario $C$, la population étant encore en 2061 plus nombreuse de $14 \%$ par rapport au niveau de 1981. Même si à terme tous les scénarios de décroissance convergent vers un point nul, 1.1 existe des différences considérables entre les chemins qui peuvent être empruntés.

I1 est intéressant de comparer le scénario $D$ avec les trois évolutions caractérisées par une fécondité qui demeure à long terme sous le seuil de remplacement. On sait que dans ce scénario la fécondité remonte pour se stabiliser à 2,1 enfants par femme. La population connaît une période d'accroissement naturel négatif, entre 2032 et 2050, mais celui-ci est modeste (pas plus de 6000 par an et 72000 seulement au total pendant la période) en comparaison des autres scénarios $(-20000$ par an dans $C$ et -55000 dans A) et est facilement compensé par un solde migratoire somme toute assez faible. La population ne diminue donc pas dans ce scénario : en 2061, elle atteint 8344000 personnes, soit presque le double du volume projeté selon le scénario $\mathrm{A}$, et $30 \%$ de plus qu'en 1981 .

Le tableau 2 permet de suivre l'évolution par bond de dix ans des composantes de 1 'accroissement de la population québécoise de 1951 à 1981 pour la période observée et jusqu'en 2061 pour les projections. On y décèle bien le rôle de l'accroissement naturel qui, positif dans les débuts, devient négatif à tour de rôle dans les quatre scénarios. L'accroissement naturel évolue sous l'action conjugée de la baisse du nombre de naissances et de la hausse du nombre de décès, résultat d'une structure par âge en vieillissement. Il est intéressant de noter qu'une différence de quelques dixièmes de point dans l'indice de fécondité entrẫne à long terme un écart somme toute assez important dans le nombre 
Tableau 2

Composantes (en milliers) de 1 'accroissement démographique du Québec, 1980-1981, et selon quatre scénarios, jusqu'à 2060-2061 (certaines années)

\begin{tabular}{|c|c|c|c|c|c|c|c|c|c|c|}
\hline Composantes & $1980-81$ & Scén. & $1990-91$ & $2000-01$ & $2010-11$ & $2020-21$ & $2030-31$ & $2040-41$ & $2050-51$ & $2060-61$ \\
\hline \multirow[t]{4}{*}{ Naissances } & 96 & A & 77 & 62 & 57 & 50 & 44 & 40 & 36 & 33 \\
\hline & & B & 92 & 76 & 74 & 70 & 64 & 62 & 59 & 56 \\
\hline & & $\mathrm{C}$ & 104 & 87 & 87 & 89 & 84 & 84 & 83 & 81 \\
\hline & & $\mathrm{D}$ & 104 & 101 & 98 & 106 & 104 & 105 & 109 & 108 \\
\hline \multirow[t]{4}{*}{ Décès } & 43 & A & 50 & 58 & 70 & 81 & 91 & 96 & 91 & 81 \\
\hline & & B & 50 & 58 & 71 & 82 & 94 & 101 & 98 & 90 \\
\hline & & $\mathrm{C}$ & 50 & 59 & 72 & 84 & 97 & 105 & 103 & 98 \\
\hline & & $\mathrm{D}$ & 50 & 59 & 73 & 88 & 102 & 111 & 110 & 105 \\
\hline \multirow{4}{*}{$\begin{array}{l}\text { Accrois- } \\
\text { sement } \\
\text { nature1 }\end{array}$} & 53 & A & 27 & 4 & -12 & -31 & -47 & -56 & -55 & -48 \\
\hline & & B & 42 & 17 & 4 & -12 & -30 & -38 & -38 & -34 \\
\hline & & $\mathrm{C}$ & 54 & 28 & 15 & 5 & -13 & -21 & -20 & -18 \\
\hline & & $\mathrm{D}$ & 54 & 42 & 26 & 19 & 2 & -6 & -1 & 3 \\
\hline \multirow{4}{*}{$\begin{array}{l}\text { Solde } \\
\text { migratoire }\end{array}$} & -1 & A & -10 & -7 & -4 & -1 & 1 & 4 & 7 & 9 \\
\hline & & B & -4 & -1 & 0 & 1 & 3 & 5 & 6 & 8 \\
\hline & & $\mathrm{C}$ & 0 & 6 & 5 & 5 & 5 & 6 & 7 & 7 \\
\hline & & $\mathrm{D}$ & 0 & 5 & 6 & 6 & 6 & 6 & 6 & 6 \\
\hline \multirow{4}{*}{$\begin{array}{l}\text { Accrois- } \\
\text { sement } \\
\text { total }\end{array}$} & 52 & A & 17 & -2 & -17 & -33 & -46 & -52 & -48 & -39 \\
\hline & & B & 38 & 16 & 4 & -11 & -27 & -34 & -32 & -26 \\
\hline & & C & 54 & 34 & 21 & 10 & -8 & -15 & -14 & -10 \\
\hline & & $\mathrm{D}$ & 54 & 47 & 32 & 25 & 8 & 0 & 5 & 9 \\
\hline
\end{tabular}

Sources : Estimations (pour 1980-81) et projections (autres années) du Bureau de la statistique du Québec. 
annuel de naissances. Ce nombre atteint 56000 et 81000 dans les scénarios $B$ et $C$ (indice de fécondité de 1,6 et 1,8 respectivement), soit un écart de 25000 naissances (une fraction de cet écart doit cependant être attribuée aux migrations plus favorables en $C$ ).

\section{$1.2 \mathrm{La}$ décroissance démographique: quelques précédents au XXe siècle}

Bien qu'il soit devenu courant pour les démographes d'élaborer des scénarios de décroissance (les scénarios de population croissante à long terme sont devenus des exceptions), nombreux sont les spécialistes (et les citoyens en généra1) qui pensent dans leur for intérieur qu'il s'agit d'une possibilité bien lointaine et même peu vraisemblable : ou la fécondité se redressera, ou les migrations viendront combler le nécessaire. Il existe pourtant des exemples de diminution de la taille d'une population au XXe siècle. Au niveau de pays entiers, si l'on excepte les périodes de guerre, la situation est encore très rare: citons tout de même la République fédérale d'Allemagne, dont la population a diminué de 663000 personnes $(-1,1 \%)$ entre 1982 et 1985 (Monnier, 1983:831 et 1985:755).

A l'intérieur de pays, l'examen de la situation est plus délicate : tout dépend des territoires considérés. Mais à ce niveau on peut trouver des exemples plus près de nous : une baisse de $17 \%$ pour 1'Ile-du-Prince-Edouard entre 1901 et 1924 , de $11 \%$ pour 1a Saskatchewan entre 1936 et 1946, de $13 \%$ pour 1 a Virginie occidentale entre 1950 et 1970. C'est au cours des années de la grande crise économique de l'entre-deux-guerres qu'il y a le plus de cas, mais il y a aussi des exemples plus récents : 1a Saskatchewan de 1968 à $1974(-6 \%)$ et l'État de New York dans la décennie soixante-dix $(-3,7 \%)$.

La population du Québec s'est continuellement accrue pendant 1 'ensemble de cette période, mais ce n'est pas le cas de toutes les régions québécoises. Ainsi, entre 1961 et 1976, la population de 1'Est du Québec a diminué de $7 \%$ et au cours de la période 1966-1971, celle de 1'Abitibi-Témiscamingue a décru de $6 \%$; 1'Ile de Montréal a vu sa population baisser de $10 \%$ entre 1971 et 1981 (Gauthier, 1977:124; BSQ, 1985:48).

I1 serait particulièrement intéressant de connâ̂tre 1a réaction des sociétés touchées par la décroissance de leur population. Le phénomène est-il perçu comme tel (menace à l'existence même du groupe) ou est-il amalgamé dans un ensemble de problèmes que l'on considère globalement? Quelles sont les réactions des porte-parole politiques, des représentants d'associations et de corps intermédiaires ? 
Nous savons cependant que dans tous les cas de décroissance démographique cités plus haut, à 1 'exception de la République fédérale d'Allemagne (où le déficit "naturel" a également joué), la source de la diminution de population doit être recherchée du côté des migrations. Ceci explique d'ailleurs qu'une fois passé la période de solde migratoire négatif, la plupart des populations en question se sont remises à crô̂tre. La différence est donc grande par rapport aux scénarios prévus pour le Québec dont la décroissance proviendrait au début du siècle prochain de $1^{\prime}$ accroissement naturel : il sera alors beaucoup moins facile de revenir à la croissance positive ou même nulle, en raison de l'effet défavorable de la structure par âge. C'est pourquoi on se tourne si aisément vers la solution migratoire.

\subsection{L'attitude à $I^{\prime}$ égard de $I^{\prime}$ immigration}

Pour éviter la décroissance, il n'existe évidemment que deux voies de solution : accrôttre la natalité ou augmenter le solde migratoire.

On connaît les difficultés que présentent les politiques natalistes : succès incertains, conflits entre les objectifs natalistes et d'autres objectifs sociaux, coûts parfois élevés. Il n'y aura probablement rien d'important qui sera entrepris de ce côté tant que l'urgence d'agir ne fera $1^{\prime}$ 'objet d'un large consensus.

En ce qui concerne la solution migratoire, elle peut être envisagée en au moins deux étapes. Dans un premier temps, on peut viser un solde migratoire nul. Ce serait une amélioration par rapport aux déficits habituels. Mais ceci ne sera pas suffisant à long terme. S'il y a insuffisance de l'accroissement naturel, il faudra recourir à une immigration d'équilibre ou compensatrice.

Dans les années soixante-dix, avec 22700 immigrants en moyenne par année, le Québec a bénéficié d'un solde international de +10 200. On est loin de ce qu'il faudrait pour équilibrer un accroissement naturel pouvant atteindre -55000 dans le scénario $A$ ou -38000 dans le scénario $B$, au milieu du siècle prochain. Par contre, c'est un niveau moins éloigné de ce qui est nécessaire dans le scénario C (accroissement naturel de -20000 environ).

Bien que le nombre d'immigrants internationaux que l'on devra accueillir annuellement dépendra avant tout du niveau de 1 'accroissement naturel, il ne faut pas oublier les autres flux migratoires. On peut supposer qu'il y aura toujours de l'émigration internationale; cette dernière devrait d'ailleurs augmenter, car l'immigration entretient l'émigration. Quant aux migrations interprovinciales, il n'est pas facile de savoir 
comment elles évolueront. Rien n'empêche le Québec de connaftre un solde positif de ce côté. Cependant, le reste du Canada sera lui aussi aux prises avec un accroissement naturel négatif et pourrait aussi être en quête d'immigrants. Il est donc raisonnable de supposer qu'au mieux le solde interprovincial sera nul. S'il est négatif, le niveau d'immigration devra compenser aussi pour ces pertes-1à. Il faut en outre supposer qu'une fois établis ici, les immigrants ont une fécondité au moins égale à celle de la moyenne québécoise, ce qui est effectivement le cas jusqu'à présent.

De tout cela, on peut conclure que le niveau d'immigration nécessaire pour compenser un éventuel déficit des naissances sur les décès, selon les conditions prévues dans les scénarios A, B ou $C$, exigera une augmentation substantielle de l'immigration. II est impossible de savoir ce que les générations futures penseront de cette immigration accrue. Cependant, nous pouvons nous interroger sur ce que pensent les Québécois du niveau actuel d'immigration. Il semble bien que la population en général soit peu favorable à l'immigration. C'est du moins ce qui ressort de 1 'examen de certains résultats tirés d'une enquête du Centre de sondage de 1'Université de Montréal. L'enquête téléphonique, menée à I'automne 1981, s'appuyait sur un échantillon d'un peu plus de 2000 personnes, représentatif de la population québécoise de 18 ans et plus. Le libellé de la question était le suivant : "Selon vous, le Québec devrait-il accueillir plus, autant ou moins d'immigrants que présentement ?".

A cette question, $60 \%$ des personnes interrogées ont répondu qu'il était préférable que le Québec en reçoive moins, $24 \%$ pensaient qu'on pouvait continuer à en accueillir autant et seulement $10 \%$ croyaient qu'il fallait accroftre l'immigration. Fait étonnant, lorsqu'on pose la même question mais au sujet des réfugiés, les réponses sont presque identiques : $55 \%$ des répondants croient qu'il faut en accueillir moins, $27 \%$ autant, et seulement $10 \%$ davantage. Soulignons qu'au moment de 1 a collecte des données, en novembre 1981, la récession ne bat pas encore son plein et le chômage bien qu'assez élevé $(10,8 \%)$ n'atteint pas encore les sommets des années 1982 à 1984. Par conséquent, si on juge que les conditions économiques ont une certaine influence sur 1 'opinion publique dans un domaine comme celui de l'immigration, 1a même question posée actuellement pourrait donner des réponses encore plus défavorables.

Voilà pour 1'opinion de 1'ensemble de la population. On peut se demander toutefois s'il n'y a pas des groupes particuliers qui manifesteraient plus de sympathie pour un maintien ou une augmentation du nombre d'immigrants au Québec. Nous avons donc fait sortir un certain nombre de résultats en fonction de diverses variables. On constate ainsi que l'attitude devient un peu plus 
favorable pour les gens très scolarisés (16 années de scolarité ou plus) ou faisant partie de ménages à revenus très élevés (50 000 \$ ou plus par année). Mais même dans ces deux catégories relativement restreintes de la population, l'attitude est davantage négative que positive : $14 \%$ des gens ayant 16 années ou plus de scolarité sont en faveur d'une augmentation du nombre d'immigrants, ainsi que $20 \%$ des gens faisant partie d'un ménage à revenu élevé, mais $35 \%$ et $34 \%$ respectivement répondent que le Québec devrait en recevoir moins. On remarque aussi que les anglophones sont davantage favorables à une augmentation de 1 'immigration ( $18 \%$ par rapport à $7 \%$ pour les francophones), mais ils sont très nombreux $(46 \%)$ à favoriser une immigration moindre. Seules les personnes de langue maternelle autre que le français ou $1^{\prime}$ anglais se prononcent en plus grande proportion pour une hausse du nombre d'immigrants ( $38 \%$, contre $29 \%$ qui sont d'opinion opposée).

Ce large consensus à l'égard de l'immigration a été confirmé par une autre enquête du Centre de sondage de l'Université de Montréal, réalisée en 1985. L'enquête portait sur l'attitude de la population devant la possibilité que la baisse de natalité n'entrấne une diminution de la population québécoise et sur les moyens à prendre pour éviter qu'une telle diminution ne se produise. Alors que $68 \%$ des gens croient qu'il faut encourager les couples à avoir plus d'enfants, seulement $11 \%$ préfèrent que le gouvernement encourage l'immigration (Proulx, 1985).

I1 faut noter qu'une attitude plutôt défavorable à l'égard de l'immigration n'est un phénomène ni nouveau ni particulier au Québec (Hoskin et Mishler, 1983). De tels résultats sont sûrement décevants pour tous ceux qui croient fermement en l'enrichissement culturel, économique et social qu'une société retire de l'immigration. Toutefois, ils mettent en garde contre les solutions ne tenant pas compte de toutes les facettes de la question. Pour que l'immigration puisse constituer un moyen d'éviter 1a décroissance numérique de la population québécoise, il faudra que cette solution reçoive un appui plus large que ce n'est le cas présentement. Il est possible que d'ici à l'avènement de la décroissance ( $i$ jamais celle-ci arrive) 1'opinion publique évolue. Quol qu'il en soit, il faut accepter que la solution de l'immigration n'offre pas plus de garantie de succès que le recours à des mesures natalistes.

2. OPERATIONNALISATION DES HYPOTHESES POUR LA POPULATION ACTIVE

\subsection{Evolution passée de la population active}

Entre 1951 et 1981, 1a population active du Québec est passée de 1472500 à 3051000 personnes. Ce doublement de volume en 
l'espace de trente ans représente un rythme annuel moyen de $2,46 \%$. Pendant 1 a même période, la population en âge de travailler ( 15 ans et plus) s'accroissait de $2,07 \%$, ce qui montre bien 1 'importance des facteurs démographiques dans 1'accroissement du nombre d'actifs. Une étude antérieure (Gauthier et Côté, 1980) a d'ailleurs permis de conclure que presque les $3 / 4$ de 1'accroissement de la population active entre 1961 et 1978 provenaient des facteurs démographiques (taille et composition par âge de la population en âge de travailler), le reste étant lié aux changements dans les taux d'activité.

Pour faire une projection du nombre d'actifs, il est nécessaire de poser au préalable des hypothèses sur les taux d'activité des années futures. Voyons comment la participation au marché du travail a varié au cours des dernières décennies. Deux sources de données nous renseignent à ce sujet : les recensements canadiens de 1951 à 1981 et l'enquête sur la population active pour les années plus récentes (1979 à 1985).

Le tableau 3 permet de se rendre compte de l'évolution des taux d'activité de 1951 à 1981. Les tendances sont très différentes pour chacun des deux sexes. Pour les hommes, I'ensemble de la période 1951-1981 est caractérisé par une baisse générale. Entre 1976 et 1981 , on note cependant une légère augmentation de leur taux d'activité (sauf chez les personnes de 55 ans et plus), comme si la baisse antérieure avait été exagérée. Il faut se méfier de la forte diminution survenue entre 1961 et 1971 qui a été suivie entre 1971 et 1976 d'une récupération presque équivalente : il semble que les questions de la version française du questionnaire aient été mal comprises parmi certains groupes de travailleurs, ce qui a pu entraîner une sous-estimation de la population active en 1971 (Kempster, 1973:2).

La dernière récession n'a pas affecté l'activité masculine de la même manière d'un groupe d'âge à l'autre : c'est ce qui découle de l'examen du tableau 4, qui porte sur les années 1979 à 1985. L'effet le plus grave a été ressenti chez les hommes âgés de 55 à 64 ans, dont le taux d'activité a chuté de $74,8 \%$ en 1980 à $69,1 \%$ en 1982 (au plus fort de la récession) et à $65,4 \%$ en 1985; une réduction de cette ampleur peut difficilement être attribuée au seul facteur de l'abaissement volontaire de l'âge de la retraite. L'effet de la récession s'est fait sentir aussi, mais à un moindre degré, dans les groupes des 20-24, 25-34 et 45-54 ans, dont les taux d'activité ont légèrement baissé. Si cette baisse de l'activité chez les hommes est d'ordre surtout conjoncturel, il est possible qu'une certaine récupération se manifeste dans 1 'avenir : la récupération est d'ailleurs sensible chez les jeunes de 15-19 ans dont le taux a augmenté entre 1982 et 1985 (de 43,7\% à $47,3 \%)$. 
Tableau 3

Taux d'activité (en \%) selon l'âge et le sexe, Québec, 1951-1981

\begin{tabular}{|c|c|c|c|c|c|c|}
\hline Groupe d'âge & 1951 & 1961 & 1971 & 1976 & 1981 & $1981^{a}$ \\
\hline & \multicolumn{6}{|c|}{ Sexe masculin } \\
\hline $15-19$ & 63,3 & 39,8 & 37,4 & 43,0 & 40,5 & 40,2 \\
\hline $20-24$ & 91,2 & 84,3 & 80,8 & 83,3 & 88,0 & 87,5 \\
\hline $25-34$ & 96,5 & 93,0 & 87,8 & 91,2 & 93,5 & 92,8 \\
\hline $35-44$ & 96,6 & 93,1 & 87,3 & 91,4 & 92,7 & 92,1 \\
\hline $45-54$ & 94,5 & 90,2 & 84,3 & 87,8 & 88,8 & 88,3 \\
\hline $55-59$ & 89,4 & $80 \Omega$ & 78,3 & 81,0 & 80,5 & 79,5 \\
\hline $60-64$ & 81,0 & 80,0 & 67,4 & 66,9 & 65,8 & 65,3 \\
\hline 65 ans et plus & 36,4 & 27,4 & 21,5 & 17,1 & 14,7 & 13,8 \\
\hline 15 ans et plus & 85,2 & 77,3 & 71,4 & 74,2 & 75,8 & 74,9 \\
\hline
\end{tabular}

Sexe féminin

\begin{tabular}{rrrrrrr}
$15-19$ & 40,5 & 38,0 & 32,5 & 36,0 & 36,6 & 36,5 \\
$20-24$ & 46,3 & 51,3 & 61,4 & 67,6 & 76,5 & 76,3 \\
$25-34$ & 23,9 & 27,0 & 39,9 & 50,9 & 61,8 & 61,6 \\
$35-44$ & 20,6 & 24,9 & 34,4 & 46,0 & 57,4 & 57,2 \\
$45-54$ & 19,1 & 26,7 & 33,8 & 40,5 & 47,6 & 47,4 \\
$55-59$ & 15,5 & 20,3 & 29,6 & 31,3 & 34,2 & 33,8 \\
$60-64$ & 12,1 & 22,6 & 22,0 & 22,8 & 22,8 \\
65 ans et plus & 5,9 & 7,2 & 9,0 & 7,7 & 5,8 & 5,3 \\
15 ans et plus & 25,1 & 28,2 & 35,0 & 41,1 & 47,5 & 46,8 \\
\hline
\end{tabular}

Sources : Recensements du Canada, 1951 (volume IV, Main-d'oeuvre, tableau 3), 1961 (bulletin 3-1.2, tableau 5), 1971 (no 94-704, tableau 9), 1976 (no 94-802, tableau 5), 1981 (no 92-915, tableau 1).

a. Taux rectifiés, tenant compte des pensionnaires d'institution. 
Tableau 4

Taux d'activité (en \%) selon 1'âge et le sexe, Québec, 1979-1985

\begin{tabular}{|c|c|c|c|c|c|c|c|}
\hline Groupe d'âge & 1979 & 1980 & 1981 & 1982 & 1983 & 1984 & 1985 \\
\hline \multicolumn{8}{|c|}{ Sexe masculin } \\
\hline $\begin{array}{l}15-19 \\
20-24 \\
25-34 \\
35-44 \\
45-54 \\
55-64 \\
65 \text { ans et plus }\end{array}$ & $\begin{array}{l}47,7 \\
84,0 \\
94,6 \\
95,0 \\
90,5 \\
73,5 \\
12,8\end{array}$ & $\begin{array}{l}50,0 \\
84,5 \\
94,3 \\
94,2 \\
90,6 \\
74,8 \\
12,3\end{array}$ & $\begin{array}{l}49,1 \\
84,2 \\
93,8 \\
94,4 \\
90,4 \\
71,6 \\
10,7\end{array}$ & $\begin{array}{l}43,7 \\
80,8 \\
91,9 \\
93,4 \\
89,2 \\
69,1 \\
11,2\end{array}$ & $\begin{array}{r}44,9 \\
80,9 \\
91,7 \\
93,2 \\
89,6 \\
69,6 \\
9,9\end{array}$ & $\begin{array}{l}46,8 \\
81,1 \\
92,0 \\
93,2 \\
88,3 \\
66,3 \\
10,0\end{array}$ & $\begin{array}{l}47,3 \\
81,3 \\
92,1 \\
98,3 \\
88,0 \\
65,4 \\
10,9\end{array}$ \\
\hline 15 ans et plus & 76,6 & 77,0 & 76,4 & 74,4 & 74,7 & 74,7 & 75,1 \\
\hline \multicolumn{8}{|c|}{ Sexe féminin } \\
\hline $\begin{array}{l}15-19 \\
20-24 \\
25-34 \\
35-44 \\
45-54 \\
55-64 \\
65 \text { ans et plus }\end{array}$ & $\begin{array}{r}42,1 \\
70,8 \\
56,4 \\
52,5 \\
43,6 \\
26,9 \\
4,2\end{array}$ & $\begin{array}{r}43,1 \\
72,3 \\
58,8 \\
54,4 \\
46,5 \\
27,5 \\
4,4\end{array}$ & $\begin{array}{r}43,0 \\
71,9 \\
62,3 \\
57,6 \\
47,1 \\
27,1 \\
4,2\end{array}$ & $\begin{array}{r}39,0 \\
71,1 \\
59,5 \\
58,4 \\
48,3 \\
26,7 \\
4,4\end{array}$ & $\begin{array}{r}40,8 \\
72,4 \\
62,6 \\
59,3 \\
48,2 \\
27,7 \\
6,0\end{array}$ & $\begin{array}{r}40,9 \\
72,5 \\
64,7 \\
63,2 \\
49,5 \\
26,2 \\
7,1\end{array}$ & $\begin{array}{r}44,0 \\
72,7 \\
67,9 \\
64,3 \\
51,3 \\
25,1 \\
4,1\end{array}$ \\
\hline 15 ans et plus & 44,6 & 46,2 & 47,4 & 46,4 & 47,9 & 49,1 & 50,0 \\
\hline
\end{tabular}

Sources : Statistique Canada, nos 71-529 et 71-001 (décembre 1984 et 1985) au catalogue. 
Chez les femmes, il y a une hausse remarquable du taux d'activité pour tous les groupes entre 20 et 60 ans, comme on peut le constater aux tableaux 3 et 4 . La hausse, stoppée par la récession de 1982, a repris depuis, particulièrement pour le groupe âgé entre 25 et 45 ans. La progression de 1'activité féminine n'aurait sans doute pu se faire si la fécondité n'avait diminué au cours des trois décennies considérées ici. Mais on peut voir au tableau 5 que la hausse de 1 'activité des Canadiennes entre 1976 et 1981 se retrouve à la fois chez celles qui n'ont pas d'enfants et chez celles qui en ont. L'augmentation chez les mères de jeunes enfants est très forte : près de la moitié sont maintenant sur le marché du travail. Une telle situation ne peut que découler d'un désir (ou un besoin...) très fort d'intégration au marché officiel du travail. Des données comparables sur l'activité des Québécoises en 1981 sont également fournies au tableau 5. On constate qu'elles participent un peu moins intensément au marché du travail que l'ensemble des Canadiennes. C'est un signe qu'il y a encore place pour des gains ultérieurs.

\subsection{Hypothèses pour le futur}

Trois possibilités ont été envisagées quant à l'évolution future des taux d'activité selon l'âge et le sexe. En voici les caractéristiques principales (voir tableau 6).

(a) Hypothèse $I$. Selon cette hypothèse, les taux d'activité de 1 'année 1985 (légèrement modifiés pour être applicables à $l^{\prime}$ 'ensemble de la population ${ }^{1}$ ) sont maintenus constants durant toute la période de projection, soit jusqu'en 2061. En gardant les taux constants pour toute la période de projection, cette simulation mettra en évidence l'effet des seuls changements démographiques.

(b) Hypothèse II. Dans la seconde hypothèse, les taux connaissent une hausse considérable, du moins pour le sexe féminin. L'activité masculine atteindrait en 1996 le niveau des taux ontariens en 1985, ce qui représenterait une légère augmentation. Une telle évolution peut se justifier dans une perspective à long terme. En ce qui concerne l'accroissement de l'activité des femmes, il n'est pas du tout facile de prévoir à quel niveau il s'arrêtera. Certains auteurs vont jusqu'à anticiper une égalisation des taux d'activité des femmes avec ceux des hommes (Le Bras et Tapinos, 1979). D'autres pensent que les taux d'activité des femmes pourraient être identiques à ceux des hommes sauf pour les groupes entre 20 et 45 ans (Ciuriak et Sims, 1980).

1. On sait que $I^{\prime}$ enquête sur la population active porte sur la population dont ont été exclus les pensionnaires d'institution, les membres des forces armées et les résidents des réserves indiennes. 
Tableau 5

Taux d'activité (en \%) des femmes (tous états matrimoniaux) par groupe d'âge, selon I'âge et la présence d'enfants, Canada, 1976 et 1981, et Québec, 1981

\begin{tabular}{|c|c|c|c|c|c|c|c|c|c|c|}
\hline \multirow[b]{3}{*}{$\begin{array}{l}\text { Groupe } \\
\text { d'âge }\end{array}$} & \multicolumn{10}{|c|}{ CANADA } \\
\hline & \multicolumn{5}{|c|}{1976} & \multicolumn{5}{|c|}{1981} \\
\hline & Total & $\begin{array}{l}\text { Sans } \\
\text { enfants }\end{array}$ & $\begin{array}{l}\text { Avec } \\
\text { enfants }\end{array}$ & $\begin{array}{c}\text { Avec } \\
\text { enfants } \\
\text { de moins } \\
\text { de } 6 \text { ans }\end{array}$ & $\begin{array}{c}\text { Avec } \\
\text { enfants } \\
\text { de } 6 \text { ans } \\
\text { et plus } \\
\text { seulement }\end{array}$ & Total & $\begin{array}{l}\text { Sans } \\
\text { enfants }\end{array}$ & $\begin{array}{l}\text { Avec } \\
\text { enfants }\end{array}$ & $\begin{array}{c}\text { Avec } \\
\text { enfants } \\
\text { de moins } \\
\text { de } 6 \text { ans }\end{array}$ & $\begin{array}{l}\text { Avec } \\
\text { enfants } \\
\text { de } 6 \text { ans } \\
\text { et plus } \\
\text { seulement }\end{array}$ \\
\hline $\begin{array}{l}20-24 \\
25-29 \\
30-34 \\
35-39 \\
40-44 \\
45-54\end{array}$ & $\begin{array}{l}68,0 \\
56,0 \\
51,5 \\
53,5 \\
54,0 \\
49,0\end{array}$ & $\begin{array}{l}79,5 \\
81,5 \\
78,5 \\
74,5 \\
68,5 \\
54,5\end{array}$ & $\begin{array}{l}36,5 \\
41,0 \\
45,0 \\
50,0 \\
51,0 \\
46,4\end{array}$ & $\begin{array}{c}36,0 \\
38,5 \\
37,5 \\
36,5 \\
35,0 \\
-\end{array}$ & $\begin{array}{l}57,4 \\
57,5 \\
56,0 \\
56,0 \\
53,5 \\
-\end{array}$ & $\begin{array}{l}77,2 \\
68,2 \\
63,3 \\
64,2 \\
64,3 \\
55,7\end{array}$ & $\begin{array}{l}87,0 \\
89,5 \\
86,5 \\
82,0 \\
75,9 \\
60,6\end{array}$ & $\begin{array}{l}45,3 \\
51,7 \\
56,2 \\
60,8 \\
62,0 \\
53,3\end{array}$ & $\begin{array}{l}44,7 \\
49,4 \\
49,5 \\
47,7 \\
43,5 \\
36,7\end{array}$ & $\begin{array}{l}63,4 \\
65,7 \\
66,0 \\
65,4 \\
63,6 \\
53,5\end{array}$ \\
\hline \multirow[t]{2}{*}{$\begin{array}{l}\text { Total } \\
15 \text { ans } \\
\text { et plus }\end{array}$} & 45,5 & 46,0 & 44,5 & 37,0 & 49,5 & 51,7 & 51,2 & 52,4 & 47,9 & 54,9 \\
\hline & & & & & & \multicolumn{5}{|c|}{ QUÉBEC 1981} \\
\hline $\begin{array}{l}20-24 \\
25-29 \\
30-34 \\
35-39 \\
40-44 \\
45-54\end{array}$ & & Non & disponib & & & $\begin{array}{l}76,5 \\
65,2 \\
58,0 \\
57,9 \\
56,5 \\
47,2\end{array}$ & $\begin{array}{l}84,8 \\
86,6 \\
83,1 \\
78,1 \\
71,5 \\
55,7\end{array}$ & $\begin{array}{l}42,6 \\
47,5 \\
49,4 \\
53,1 \\
52,8 \\
43,4\end{array}$ & $\begin{array}{l}42,2 \\
46,6 \\
45,4 \\
43,5 \\
38,3 \\
32,4\end{array}$ & $\begin{array}{l}51,9 \\
55,0 \\
56,6 \\
56,9 \\
54,2 \\
43,5\end{array}$ \\
\hline $\begin{array}{l}\text { Total } \\
15 \text { ans } \\
\text { et plus }\end{array}$ & & & & & & 47,4 & 49,9 & 44,5 & 44,6 & 44,4 \\
\hline
\end{tabular}

a. Peut avoir aussi des enfants de 6 ans et plus. 
Tableau 6

Trois hypothèses de taux d'activité (en \%) selon l'âge et le sexe

\begin{tabular}{|c|c|c|c|c|c|c|c|c|c|c|c|}
\hline \multirow{2}{*}{$\begin{array}{l}\text { Groupe } \\
\text { d'âge }\end{array}$} & \multicolumn{2}{|c|}{ Нyро. I } & \multicolumn{2}{|c|}{$\begin{array}{l}\text { Hypo.II: } 1986 \\
\text { Hypo.III: } 1991\end{array}$} & \multicolumn{2}{|c|}{$\begin{array}{l}\text { Нyрo.II: } 1991 \\
\text { Нypo.III: } 2001\end{array}$} & \multicolumn{2}{|c|}{$\begin{array}{l}\text { Hypo.II: } 1996 \\
\text { Нypo.III: } 2011\end{array}$} & \multirow{2}{*}{$\frac{\text { II : } 2001}{\text { III }: 2021}$} & \multirow{2}{*}{$\begin{array}{r}\text { II : } 2006 \\
\text { III }: 2031 \\
F\end{array}$} & \multirow{2}{*}{$\frac{\text { II : } 2011}{\text { III: } 2041}$} \\
\hline & $\mathrm{H}$ & F & $\mathrm{H}$ & F & $\mathrm{H}$ & F & $\mathrm{H}$ & F & & & \\
\hline $15-19$ & 46,2 & 43,6 & 46 & 45 & 52 & 50 & 57 & 55 & 55 & 55 & 55 \\
\hline $20-24$ & 79,4 & 72,0 & 79 & 74 & 82 & 76 & 84 & 78 & 78 & 78 & 78 \\
\hline $25-34$ & 91,0 & 67,5 & 91 & 68 & 93 & 72 & 95 & 76 & 80 & 80 & 80 \\
\hline $35-44$ & 92,9 & 63,9 & 93 & 64 & 94 & 70 & 96 & 76 & 78 & 78 & 78 \\
\hline $45-54$ & 87,2 & 51,0 & 87 & 52 & 90 & 62 & 93 & 69 & 76 & 76 & 76 \\
\hline $55-64$ & 64,7 & 24,8 & 65 & 26 & 69 & 35 & 73 & 41 & 47 & 53 & 60 \\
\hline $\begin{array}{l}65 \text { ans } \\
\text { et plus }\end{array}$ & 10,3 & 3,7 & 10 & 6 & 11 & 7 & 12 & 8 & 8 & 8 & 8 \\
\hline
\end{tabular}

Note : Les sigles $\mathrm{H}$ et $\mathrm{F}$ désignent respectivement les sexes masculin et féminin. 
Tableau 7

Taux d'activité (en \%) pour deux groupes de femmes ayant fait des études universitaires, par groupe d'âge et selon $1 a$ présence d'enfants, Québec, 1981

\begin{tabular}{|c|c|c|c|c|c|c|}
\hline \multirow{2}{*}{$\begin{array}{l}\text { Groupe } \\
\text { d'âge }\end{array}$} & \multicolumn{3}{|c|}{$\begin{array}{l}\text { Etudes universitaires ou } \\
\text { autres études non univ. } \\
\text { avec certificat ou dipjôme }\end{array}$} & \multicolumn{3}{|c|}{ Grade universitaire } \\
\hline & Tota1 & $\begin{array}{l}\text { Sans } \\
\text { enfants }\end{array}$ & $\begin{array}{l}\text { Avec } \\
\text { enfants }\end{array}$ & Total & $\begin{array}{l}\text { Sans } \\
\text { enfants }\end{array}$ & $\begin{array}{l}\text { Avec } \\
\text { enfants }\end{array}$ \\
\hline $20-24$ & 85,8 & 87,3 & 63,5 & 83,1 & 84,6 & 62,9 \\
\hline $25-29$ & 81,5 & 93,3 & 65,8 & 87,1 & 92,5 & 71,9 \\
\hline $30-34$ & 73,5 & 92,3 & 65,0 & 80,7 & 92,4 & 71,5 \\
\hline $35-39$ & 73,3 & 90,4 & 68,6 & 82,7 & 93,7 & 77,1 \\
\hline $40-44$ & 73,7 & 89,0 & 69,4 & 84,1 & 92,8 & 79,0 \\
\hline $45-54$ & 69,1 & 79,5 & 63,9 & 83,3 & 89,7 & 77,0 \\
\hline
\end{tabular}

Source: Statistique Canada, Recensement de 1981, tableau SPE81B11 sur microfiche.

Quant à nous, nous supposerons qu'à long terme l'ensemble des femmes tendraient à adopter le comportement des sous-groupes féminins les plus présents sur le marché du travail. On sait que la participation des femmes au marché du travail augmente avec le niveau de scolarité. On constate d'allleurs que le taux d'activité des deux groupes de Québécoises les plus scolarisées (tableau 7) est beaucoup plus élevé que celui de l'ensemble des Québécoises (tableau 5), chez les femmes sans enfants comme chez celles qui en ont. Nous allons donc retenir comme hypothèse un taux d'activité intermédiaire entre ceux des deux groupes de femmes les plus scolarisées.

Un tel niveau d'activité pourrait être atteint par l'ensemble des femmes des trois groupes d'âge entre 25 et 55 ans, au terme d'une progression qui respecterait l'évolution antérieure des générations féminines. Les données des recensements permettent de suivre 1'activité de groupes de générations : les courbes en trait plein du graphique 1 illustrent l'évolution observée, alors que les courbes en pointillé constituent l'évolution prévue des 
groupes de 25 à 55 ans. Cette méthode a permis d'obtenir les taux des années 1991 et 2001; les taux pour 1'année 1986 et 1996 ont été calculés par interpolation. Pour le groupe de 55 à 64 ans, nous prévoyons une augmentation extrêmement forte de l'activité : une telle évolution peut être envisagée du fait de la progression observée ou prévue aux âges antérieurs dans les générations concernées. Pour ce groupe, il est même nécessaire de poursuivre 1 'augmentation jusqu'en 2011 de façon à respecter l'évolution au niveau des générations. Chez les plus jeunes, nous avons supposé une forte hausse du taux qui à long terme pourrait presque rejoindre le taux ontarien de 1985. Finalement, les taux dans les groupes de 20 à 24 ans et de 65 ans et plus s'accroîtraient légèrement. Le tableau 6 reproduit l'évolution prévue pour les femmes selon 1 'hpothèse II.

I1 est certain que la forte hausse du taux des femmes âgées de 55 à 64 ans peut paraître étonnante. Une analyse de type transversal n'aurait pas conduit à une telle évolution : les taux des groupes de 55-59 ans et 60-64 ans n'augmentent que très peu entre 1971 et 1981 (voir tableau 3), contrairement à ce qui se passe pour les femmes de moins de 55 ans. Par contre, si l'on pratique une analyse longitudinale, il est impossible de ne pas envisager une augmentation appréciable de la participation au marché du travail des femmes du groupe 54-64 ans. En fait, ces femmes ne feront que conserver $1^{\prime}$ emploi qu'elles ont détenu à un âge antérieur. Reste à savoir si l'économie rendra possible une hausse générale de l'activité féminine.

(c) Hypothèse III. Cette troisième hypothèse est en fait une variante de la deuxième : les taux d'activité atteindraient un

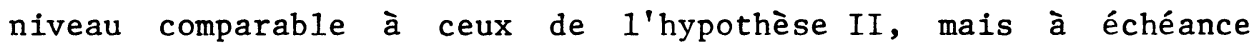
beaucoup plus lointaine. Le niveau de l'activité se stabiliserait en 2011 pour les hommes et en 2041 pour les femmes, au niveau atteint dès 1996 et 2011, respectivement, dans 1'hypothèse II. Une telle évolution des taux d'activité permettra d'examiner dans quelle mesure on peut retarder l'avènement de la décroissance de la population active.

Il faut noter que le choix de ces hypothèses a été fait dans le but de répondre à certaines interrogations concernant les incidences de l'évolution démographique prévue. Il est évident que d'autres possibilités existent. Par exemple, on aurait pu envisager que la hausse de l'activité des femmes se produise en même temps qu'une baisse de l'activité masculine. Un tel scénario est intéressant du point de vue de la composition par sexe de la population active (forte augmentation de la proportion de femmes), mais il apporte peu quant aux préoccupations ayant suscité cet article : l'effet de chacun de ces deux mouvements sur le volume de la population active s'annulerait en grande partie. 


\section{Graphique 1}

Taux d'activité (en \%) selon 1'âge, par groupe de générations féminines, Québec

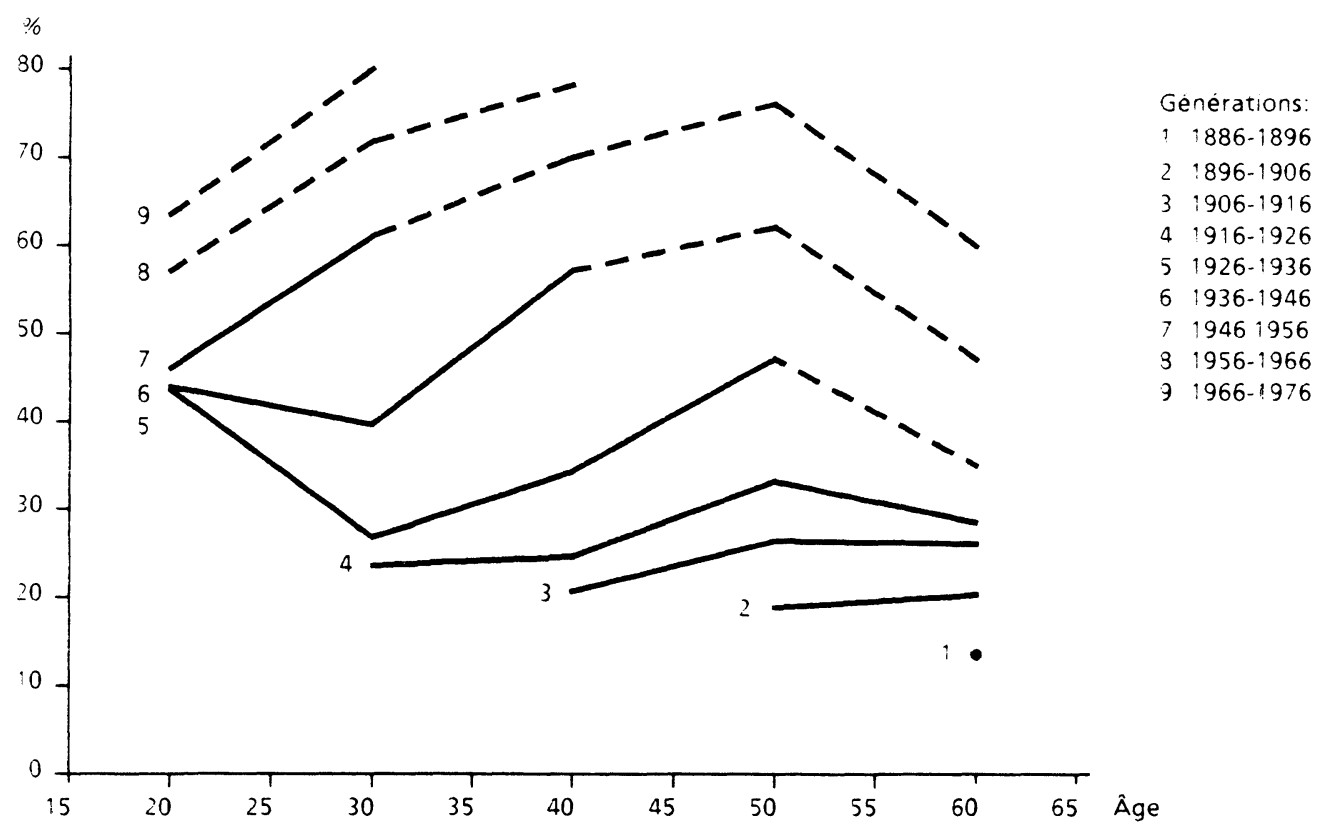

Sources : Recensements du Canada et hypothèses de 1'auteur.

\section{PRÉSENTATION DES RÉSULTATS}

Les résultats concernant l'évolution prévue de la population active seront commentés sous quatre aspects : le nombre d'actifs, la composition par sexe et par âge, le taux brut d'activité et le poids relatif des jeunes et des âgés (par rapport au nombre d'actifs).

\subsection{Effectifs totaux de la population active}

Le graphique 2 reproduit les résultats obtenus pour chacune des trois hypothèses d'activité. L'enquête sur la population active de Statistique Canada fournit la situation de départ en 1985 : le nombre d'actifs québécois est estimé pour cette année-là à 3181000 . De ces courbes, il se dégage un modèle général d'évolution du nombre d'actifs : celui-ci augmente pendant deux ou trois décennies, puis il décrôt jusqu'à la fin de la période de projection. Le niveau varie d'un scénario démographique à 
Evolution de la taille de la population active selon quatre scénarios démographiques et trois hypothèses d'activité,

\section{Millions}

Québec, 1981-2061
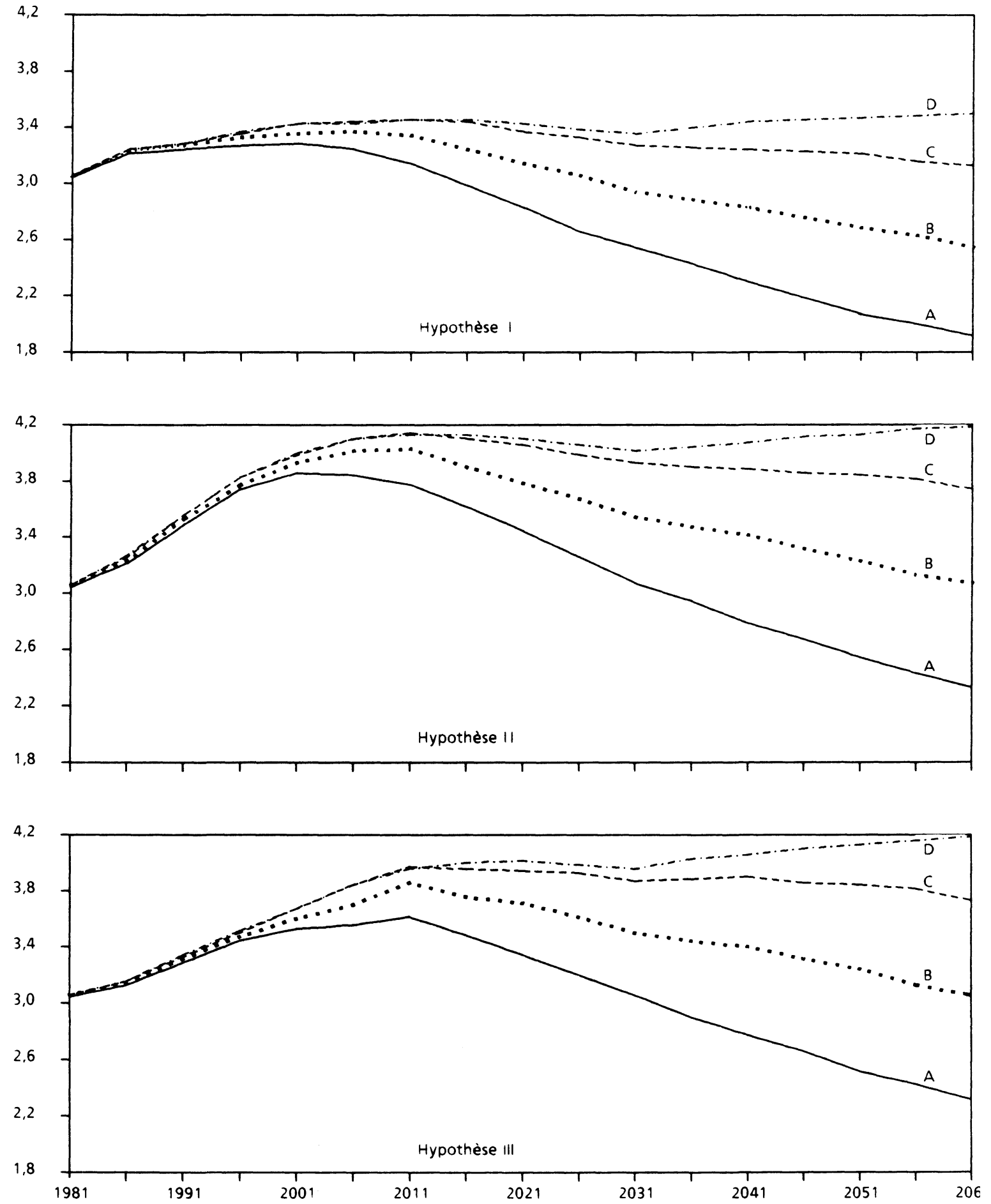
1'autre, majs les deux étapes de l'évolution sont suivies dans tous les cas, à $I^{\prime}$ exception du scénario D. Dans ce cas, il faut ajouter une troisième phase, car la hausse reprend après 2031. Cette similitude dans les diverses évolutions découlent évidemment des caractéristiques de la population de départ, dans laquelle il y a un potentiel d'accroissement de la population en âge de travailler, et des hypothèses retenues pour les projections démographiques, qui évoluent vers des niveaux constants. Notre propos n'est pas ici de discuter de la pertinence de cette façon de procéder, mais plutôt d'indiquer les incidences entraînées par diverses conditions dont il apparaît intéressant de connaître les aboutissements.

Malgré l'existence d'un schéma d'évolution générale semblable, les divergences sont grandes entre les diverses situations envisagées ici. Considérons au tableau 8 les variations dues aux seuls changements démographiques (c'est-à-dire pour un jeu d'hypothèses d'activité donné). Le nombre d'actifs obtenu en 2061 varie de façon sensible : en $D$, il surpasse de $81 \%$ ( $83 \%$ avec 1 'hypothèse d'activité I) ce que $I^{\prime}$ on trouve en $A$. Même si $I^{\prime}$ on compare deux évolutions démographiques plus rapprochées 1 'une de 1'autre, comme A (scénario "faible») et B (scénario "moyen"), les différences sont grandes, puisqu'il y a un tiers d'actifs de plus dans le second scénario. Lorsqu'on considère le très long terme, tout écart dans les hypothèses démographiques acquiert un poids considérable.

Tableau 8

Nombre d'actifs le plus élevé atteint dans chaque scénario démographique et nombre en 2061 selon I'hypothèse d'activité

\begin{tabular}{|c|c|c|c|c|c|}
\hline \multicolumn{2}{|c|}{$\begin{array}{l}\text { Hypothèse } \\
d^{\prime} \text { activité }\end{array}$} & A & B & $\mathrm{C}$ & D \\
\hline I & maximum & $\begin{array}{c}3299476 \\
(2001)\end{array}$ & $\begin{array}{c}3384203 \\
(2006)\end{array}$ & $\begin{array}{c}3480533 \\
(2011)\end{array}$ & \\
\hline & 2061 & 1924022 & 2568607 & $3 \quad 150 \quad 501$ & 3521381 \\
\hline II & maximum & $\begin{array}{c}3853051 \\
(2001)\end{array}$ & $\begin{array}{c}4013042 \\
(2011)\end{array}$ & $\begin{array}{c}4166827 \\
(2011)\end{array}$ & \\
\hline & 2061 & 2322442 & $\begin{array}{lll}3 & 081 & 149\end{array}$ & 3764497 & 4195691 \\
\hline III & maximum & $\begin{array}{c}3628035 \\
(2011)\end{array}$ & $\begin{array}{c}3847358 \\
(2011)\end{array}$ & $\begin{array}{c}4012358 \\
(2016)\end{array}$ & \\
\hline & 2061 & 2322442 & 3081149 & 3764497 & 4195691 \\
\hline
\end{tabular}


L'effet de I'hypothèse d'activité est important aussi. Pour chacun des scénarios démographiques, la population active obtenue avec 1 'hypothèse II est supérieure de 19 à $21 \%$ à ce qu'elle est en appliquant 1 'hypothèse $I$. L'effet est donc moindre que celui décelé entre les différents scénarios démographiques.

L'ampleur de la décroissance est très variable d'un scénario à l'autre. Si on calcule le pourcentage de diminution du nombre d'actifs en prenant comme base le nombre le plus élevé atteint dans chacun des scénarios, on obtient les chiffres suivants (hypothèse d'activité I) : $-42 \%$ (A), $-24 \%$ (B) et $-9 \%$ (C). Si la baisse qui s'étale sur une cinquantaine d'années est plutôt faible dans le scénario $C$, ce n'est pas le cas en $A$ puisqu'elle toucherait presque la moitié de l'effectif; dans ce dernier cas, $l^{\prime}$ effet sur la structure productive serait sûrement majeur.

Il est intéressant de comparer à quelle période la décroissance survient. Avec 1 'hypothèse d'activité $I$, ce phénomène se produit à partir de 2001 (A), 2006 (B) ou 2011 (C) : l'évolution de la population active suit le même cheminement que la population en âge de travailler qui commence aussi à décroître à partir des mêmes périodes. On constate que l'hypothèse d'activité II ne modifie la période du début de la décroissance que dans le cas du scénario B (début après 2011 au lieu de 2006). Avec I'hypothèse d'activité III, dans laquelle la hausse de 1 'activité s'étend sur une plus longue période, la décroissance est retardée d'une décennie dans le cas du scénario A (début en 2011 au lieu de 2001) et de cinq ans pour le scénario C (2016 plutôt que 2011). Le sursis dans 1 'avènement de la décroissance que permet d'introduire le jeu de l'activité n'est pas de longue durée : dix ans (scénario A) ou cinq ans (scénarios B et $C$ ). On peut qualifier cet effet de modeste, compte tenu de l'importance des changements dans l'intensité de l'activité impliqués dans les hypothèses II et III $^{2}$.

Par ailleurs, on sait qu'avec les hypothèses d'activité II et III le potentiel d'activité a été fortement accru. On est alors à même de saisir tout le poids que les variables démographiques ont dans l'évolution de la taille de la population active : Ie potentiel de décroissance impliqué par l'évolution de la population en âge de travailler n'est pas du tout épuisé en 2061 dans les trois scénarios $A, B$ et $C$, alors que le potentiel d'augmentation de $I^{\prime}$ activité est presque exploité à fond. A la limite, on pourrait sans doute tendre à l'égalité entre population

2. La précision aurait évidemment été plus grande si nous avions fait les projections année par année; les conclusions ne peuvent cependant guère en être modifiées de façon sensible. 
en âge de travailler et population active, mais il s'agit là d'une possibilité bien peu réaliste. L'augmentation des taux d'activité n'est donc qu'un bien mince recours si on cherche à contrer d'une façon durable la décroissance de la population active.

I1 semble en outre intéressant de signaler que la décroissance du nombre d'actifs pourrait survenir avant celle de la population totale. C'est ce qui se produit dans le cas de l'hypothèse d'activité $I$ avec les scénarios $B$ et $C$. Une telle éventualité pourrait avoir des conséquences importantes. En effet, il est possible d'imaginer que dans un tel cas des mesures prises pour contrecarrer le déclin du nombre d'actifs, par 1'accroissement de l'immigration par exemple, auraient comme résultat d'empêcher le déclin ultérieur de la population totale.

\subsection{Composition par sexe et par âge de la population active}

Etant donné la masse de données, il n'est évidemment pas possible, dans le cadre d'un article, de discuter des résultats par âge et sexe obtenus pour chaque combinaison entre scénario démographique et hypothèse d'activité. Nous ne retiendrons pour l'essentiel que ceux relatifs au scénario B ("moyen"), caractérisé par un solde migratoire "moyen» et une fécondité en légère reprise.

Il n'est guère étonnant de constater que la proportion de femmes parmi les actifs augmente de $41,3 \%$ en 1985 à $45,1 \%$ en 2001 (pour se maintenir autour de $45,5 \%$ par la suite) dans le cas de 1'hypothèse II (qui suppose une hausse de la participation féminine au marché du travail). L'augmentation de la part des femmes dans la population active est assez forte si $1^{\prime}$ on tient compte du fait que les changements dans la structure démographique ont un effet contraire. La proportion de femmes diminue en effet de $41,3 \%$ à $40,1 \%$ en 2001 et à environ $39 \%$ par la suite lorsque, en maintenant les taux d'activité constants (hypothèse $I$ ), on ne fait jouer que les variations démographiques; il en est ainsi parce que 1'écart dans 1'activité avant et après 45 ans étant beaucoup plus marqué chez les femmes que chez les hommes (voir le tableau 6), le vieillissement de la population en âge de travailler a un effet plus défavorable dans leur cas. Pour l'ensemble du Canada, Denton, Feaver et Spencer (1980:40, tableau 6-4) prévoient à long terme entre $38 \%$ et $42 \%$ de femmes dans la population active, ce qui dénote des hypothèses d'activité féminine plutôt conservatrices par rapport aux nôtres.

Le vielllissement de la population en âge de travailler va évidemment se répercuter sur la population active. Le vieillissement de celle-ci est manifeste au tableau 9 qui retient 
Tableau 9

Répartition (en \%) par âge de la population active en 1981 et 1985 et selon le scénario démographique $B$ et 1 'hypothèse d'activité I de 1991 à 2061

\begin{tabular}{lrrrrrrrrrr}
\hline $\begin{array}{l}\text { Groupe } \\
\text { d'âge }\end{array}$ & 1981 & 1985 & 1991 & 1996 & 2001 & 2011 & 2021 & 2031 & 2061 & $\begin{array}{r}2061 \\
\text { H.II }\end{array}$ \\
\hline $15-24$ & 25,0 & 21,9 & 17,0 & 16,3 & 16,5 & 16,4 & 15,0 & 15,6 & 15,5 & 14,7 \\
$25-34$ & 29,4 & 30,0 & 30,2 & 26,1 & 22,1 & 22,4 & 23,4 & 22,1 & 22,5 & 20,7 \\
$35-44$ & 21,2 & 23,7 & 26,5 & 28,1 & 28,6 & 22,2 & 23,7 & 24,8 & 24,2 & 22,3 \\
$45-54$ & 15,3 & 14,7 & 16,6 & 19,6 & 21,6 & 24,2 & 20,0 & 21,5 & 21,5 & 21,9 \\
$55-64$ & 8,0 & 8,3 & 8,3 & 8,3 & 9,5 & 12,8 & 15,1 & 12,6 & 12,9 & 16,2 \\
65 et & 1,2 & 1,3 & 1,5 & 1,6 & 1,7 & 2,0 & 2,8 & 3,5 & 3,4 & 4,2 \\
plus & 1,2 & & & & & & & & & \\
Total & 100,0 & 100,0 & 100,0 & 100,0 & 100,0 & 100,0 & 100,0 & 100,0 & 100,0 & 100,0 \\
\hline
\end{tabular}

I'hypothèse d'activité I (taux d'activité de 1985 maintenus constants) appliquée au scénario B (Hmoyen"). Ainsi, 1a proportion des actifs de 45 ans et plus passe de $24 \%$ en 1985 à $33 \%$ en 2001 et elle atteint $38 \%$ en 2031. C'est le groupe d'actifs de moins de 35 ans qui subit toute la baisse. Nous avons ajouté l'année 1981 au tableau pour bien montrer la rapidité des changements en cours : les jeunes actifs (15-24 ans) volent leur proportion diminuer de $25,0 \%$ à $21,9 \%$ entre 1981 et 1985 , et ils ne représenteraient plus que $17,0 \%$ en 1991 . La baisse de 8 points de pourcentage en une décennie est la conséquence directe de 1 'arrivée à 1 'âge actif des générations moins nombreuses nées à partir de 1966. Le groupe âgé de 25 à 34 ans sera touché 10 ans plus tard, soit après 1991 : sa proportion chute de $30 \%$ à $22 \%$ dans la dernière décennie du siècle.

Dans le cas d'une augmentation des taux d'activité (hypothèse II - dernière colonne du tableau 9), le vieillissement de la population active est encore plus accentué du fait notamment de la 
forte hausse de l'activité féminine après 45 ans : en 2061, la proportion d'actifs de 45 ans et plus serait de $37,8 \%$ ou $42,3 \%$, selon que $1^{\prime}$ on garde les taux d'activité constants ou qu'on les fasse augmenter. Par rapport à la situation actuelle $(24,3 \%$ de 45 ans et plus), le vieillissement des actifs attribuable aux changements dans la structure démographique est donc beaucoup plus important que celui découlant de changements dans l'activité, du moins à 1 'intérieur des normes que nous avons posées au préalable.

\subsection{Evolution du taux brut d'activité}

Le taux brut d'activité représente le nombre d'actifs en pourcentage de la population totale. Il diffère du taux d'activité habituellement utilisé qui ne se réfère qu'à la population en âge de travailler, soit les 15 ans et plus. Le taux brut d'activité est un indice relativement simple mais 11 n'en est pas moins important. En effet, comme ce sont les actifs qui assurent la production de biens et services, le taux brut d'activité peut fournir une indication du niveau de "bien-être" de la population.

Pour un niveau d'activité donné (en l'occurrence celui de 1985), on dénote très peu de variation dans le taux brut d'activité entre les scénarios démographiques (tableau 10). L'écart maximum se situe vers 2011 , mais il est faible ( 3 points de pourcentage). En 2031, comme en 2061, il n'y a presque plus d'écart entre scénarios. Cela tient au fait que la proportion de personnes en âge de travailler varie peu d'un scénario à l'autre. Par contre, la sensibilité du taux brut d'activité est plus grande à la variation de la propension à travailler : 1'écart entre les hypothèses I et II se situe entre 8 et 9 actifs pour 100 personnes de la population totale. Le graphique 3 illustre clairement ce phénomène. Notons cependant qu'à long terme, il n'y a pas de différence entre les hypothèses II et III.

I1 est intéressant de noter que 1'hypothèse d'activité II (hausse rapide des taux d'activité) fait qu'à terme la proportion d'actifs dans la population totale (51,5\% dans le scénario démographique B) est inférieure au maximum atteint dans les années 1996-2021 (autour de $56 \%$ ), mais elle demeure supérieure au niveau de $1985(48,3 \%)$. L'hypothèse I (taux de 1985 gardés constants) montre bien que les variations démographiques dans la structure par âge conduisent à une réduction du taux brut d'activité (taux brut d'activité de $42,9 \%$ en 2061 dans le cas du scénario B). Du point de vue démographique, la période actuelle (jusque vers 2011) apparaît favorable. Une telle conclusion est encore plus vraie si on considère l'évolution du rapport de dépendance économique, notamment en ce qui concerne le poids relatif des personnes âgées. 
Tableau 10

Taux brut d'activité ${ }^{a}$ dans le cas de I'hypothèse d'activité I selon quatre scénarios démographiques, 1985-2061

\begin{tabular}{lcccc}
\hline Année & A & B & C & D \\
\hline 1985 & 48,3 & 48,3 & 48,3 & 48,3 \\
1991 & 48,7 & 48,1 & 47,9 & 47,9 \\
2001 & 49,0 & 47,7 & 46,9 & 46,9 \\
2011 & 47,5 & 46,8 & 46,1 & 44,6 \\
2021 & 44,5 & 44,3 & 43,9 & 42,5 \\
2031 & 42,5 & 42,8 & 42,6 & 41,0 \\
2061 & 42,3 & 42,9 & 43,1 & 42,2 \\
\hline
\end{tabular}

a. Nombre d'actifs pour 100 personnes dans la population totale.

\section{Graphique 3}

Evolution du taux brut d'activité dans le cas du scénario "moyen"

(B) selon trois hypothèses d'activité. Québec, 1985-2061

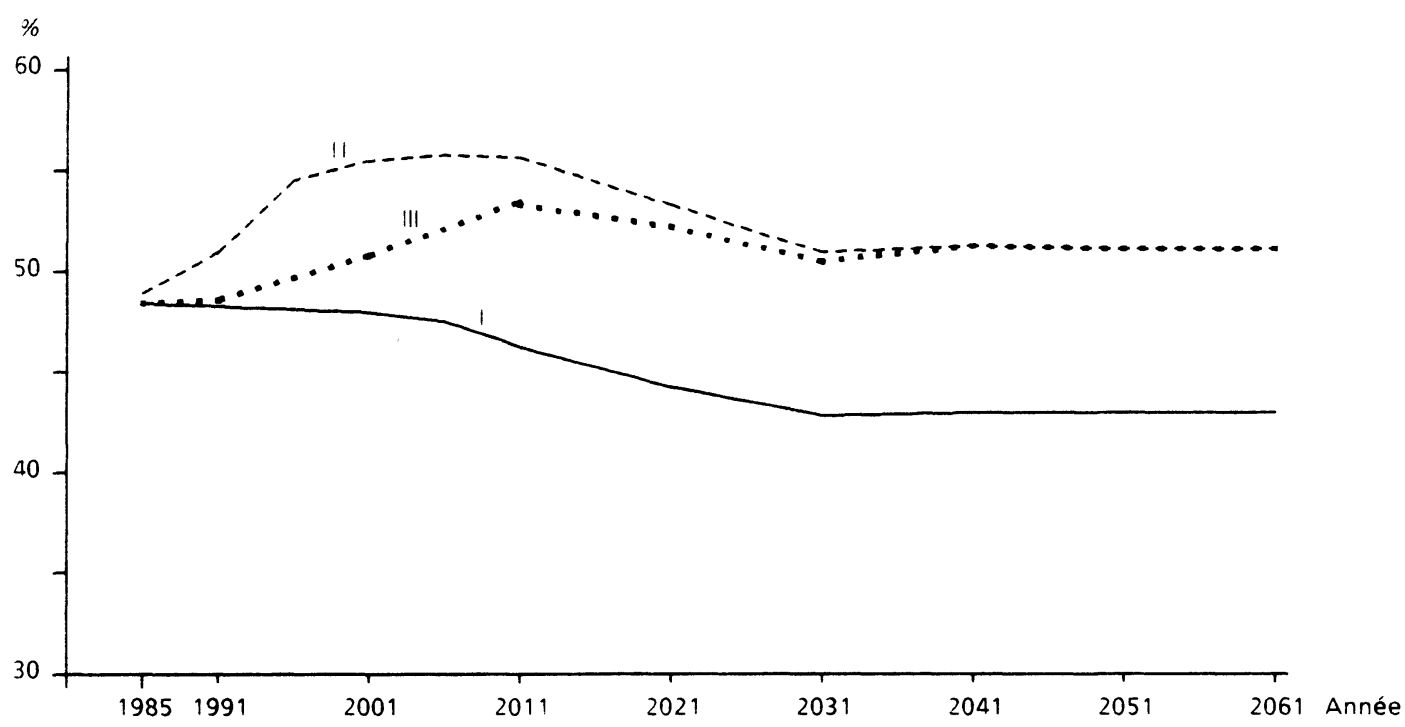




\subsection{Evolution du rapport de dépendance économique}

Le rapport de dépendance économique représente le nombre de jeunes et de personnes âgées pour 100 actifs. Il diffère donc par son dénominateur du rapport de dépendance démographique qui s'établit pour 100 personnes de 15-64 ans. A très long terme, il faut s'attendre à trouver un rapport de dépendance économique très semblable entre les scénarios démographiques (c'est ce que $1^{\prime}$ on observe au tableau 11), bien qu'il existe une période transitoire au cours de laquelle une structure est d'autant plus avantageuse que la fécondité est faible. Les deux types de rapport de dépendance évoluent en parallèle comme on le constate en comparant leur évolution pour le scénario B.

\section{Tableau 11}

Rapport de dépendance économique ${ }^{a}$ selon quatre scénarips démographiques et rapport de dépendance démographique pour le scénario B, 1985-2061

\begin{tabular}{|c|c|c|c|c|c|c|}
\hline \multirow{3}{*}{ Année } & \multicolumn{5}{|c|}{ RAPPORT DE DEPENDANCE ECONOMIQUE ${ }^{a}$} & \multirow{3}{*}{$\begin{array}{c}\text { RAPPORT DE } \\
\text { DEPENDANCE } \\
\text { DEMOGRAPHIQUE } \\
\mathrm{B}\end{array}$} \\
\hline & \multicolumn{3}{|c|}{ Hypothèse I } & & \multirow{2}{*}{$\frac{\text { Hyp. II }}{\text { B }}$} & \\
\hline & $\mathrm{A}$ & B & $\mathrm{C}$ & $\mathrm{D}$ & & \\
\hline 1985 & 63 & 63 & 63 & 63 & 63 & 44 \\
\hline 1991 & 63 & 65 & 66 & 66 & 61 & 46 \\
\hline 2001 & 60 & 65 & 69 & 72 & 56 & 45 \\
\hline 2011 & 63 & 66 & 69 & 76 & 55 & 45 \\
\hline 2021 & 77 & 78 & 80 & 87 & 65 & 53 \\
\hline 2031 & 90 & 89 & 90 & 100 & 74 & 62 \\
\hline 2061 & 91 & 88 & 87 & 92 & 73 & 61 \\
\hline
\end{tabular}

$$
\begin{aligned}
& \text { a. } \frac{(0-14 \text { ans })+(65 \text { ans et plus }) \times 100}{\text { nombre d'actifs }} \\
& \text { b. } \frac{(0-14 \text { ans })+(65 \text { ans et plus }) \times 100}{(15-64 \text { ans })}
\end{aligned}
$$


On peut se demander dans quelle mesure l'augmentation du taux d'activité pourrait compenser la hausse progressive du rapport de dépendance au siècle prochain. Si la propension à travailler évolue selon 1 'hypothèse II, il y a effectivement un certain ralentissement dans la croissance du rapport de dépendance; celui-ci demeure cependant supérieur à ce qu'il était en 1985 (73 par rapport à 63).

Mais au delà de l'évolution du nombre total de dépendants, il faut également considérer les changements prévus dans la composition de ces dépendants. Prenons par exemple le scénario "moyen" (B). Le tableau 12 montre que non seulement y a-t-il une augmentation du rapport de dépendance économique, de 63 à 88 pour 100 actifs, mais la nature des dépendants est profondément modifiée. La portion des dépendants jeunes diminue beaucoup moins entre 1985 et 2061 que n'augmente 1a portion des dépendants âgés : celle-ci double puisqu'elle passe de $32 \%$ à $60 \%$ des dépendants. La question principale n'est donc pas tellement l'augmentation du rapport de dépendance, mais bien quelle est la charge d'un jeune par rapport à celle d'une personne âgée.

Le même type de problème se pose lorsqu'on compare les scénarios démographiques sous cet aspect. En effet, on a vu que le rapport de dépendance économique est presque identique entre les quatres scénarios (variations de 87 à 92 dépendants pour 100 actifs). Mais alors que dans le scénario A, le tiers seulement de ces dépendants sont des jeunes ( 29 sur 91 ), il y en a la moitié dans le scénario $D$ (46 sur 92). Au problème de l'importance numérique des dépendants s'ajoute celui peut-être plus crucial de leurs caractéristiques qualitatives.

\section{Tableau 12}

Evolution du nombre de jeunes et du nombre de personnes âgées pour 100 actifs, dans le cas de 1 'hypothèse d'activité I, 1985-2061

\begin{tabular}{lcccc}
\hline Année & Scénario & $\begin{array}{c}\text { Nombre de } \\
\text { jeunes }(0-14 \text { ans })\end{array}$ & $\begin{array}{c}\text { Nombre âgés } \\
(65+)\end{array}$ & $\begin{array}{c}\text { Total } \\
\text { dépendance économique }\end{array}$ \\
\hline 1985 & B & 43 & 20 & 63 \\
1991 & B & 42 & 23 & 65 \\
2001 & B & 38 & 27 & 65 \\
2011 & B & 34 & 32 & 66 \\
2021 & B & 35 & 43 & 78 \\
2031 & B & 35 & 54 & 89 \\
2061 & B & 35 & 53 & \\
& & & & 91 \\
2061 & A & 29 & 62 & 87 \\
2061 & C & 39 & 48 & 92 \\
2061 & D & 46 & 46 & \\
\hline
\end{tabular}




\section{CONCLUSION}

Sur le plan de l'évolution des structures, on arrive à la conclusion qu'il y a peu de variations à terme entre les différents scénarios démographiques. Cela est vrai notamment pour le taux brut d'activité et le rapport de dépendance économique. Une transformation majeure est cependant à signaler en ce qui concerne la composition des dépendants : la population âgée y prend un poids de plus en plus grand. La question essentielle devient alors ce que représente la charge d'un jeune par rapport à celle d'une personne âgée. Il y a peu d'études à ce sujet (voir cependant McDonald, 1978), et toute affirmation fondée sur les données actuelles risque d'être démentie au gré des modifications dans les programmes gouvernementaux, nombreuses ces années-ci.

Autre changement de structure pour lequel il est difficile de porter un jugement catégorique : la population active va vieillir, et elle va vieillir d'autant plus que la fécondité sera faible. Cela signifie plus d'expérience et de maturité, mais moins de mobilité et peut-être moins de dynamisme. Certains pensent que, grâce à des mesures institutionnelles et à divers mécanismes sociaux, on pourra corriger plus ou moins facilement les effets négatifs découlant du vieillissement de la population active et notamment sa plus faible mobilité, mais d'autres (Wander, 1978:101) évoquent la possibilité que les obstacles institutionnels viennent plutôt aggraver les problèmes posés par le déclin du nombre d'actifs.

Si l'évolution des structures est importante, il ne faut pas négliger pour autant celle des effectifs. Un déclin des effectifs sur une très longue période aurait sûrement des conséquences autrement plus sérieuses que les modifications de structure. Lorsqu'on examine l'évolution de la taille de la population (totale ou active), la comparaison entre différents scénarios démographiques tourne clairement au désavantage des scénarios dont la fécondité est plus faible. Dans les trois scénarios (A, B et C) où la fécondité est sous le seuil de remplacement des générations, la décroissance du nombre d'actifs pourrait survenir dans un avenir qui n'est somme toute pas très éloigné : entre 15 et 25 ans. Des hypothèses d'activité plus favorables peuvent reculer à 25 ans environ l'échéance la plus rapprochée. Le relèvement de l'activité ne modifie donc pas de façon radicale les données du problème de la décroissance éventuelle de la population active. 
Au début du siècle, dans L'indépendance économique du Canada francais, Errol Bouchette (1906) écrivait : "Le nombre est utile pour le présent, la qualité est essentielle pour l'avenir". Au cours des huit décennies écoulées depuis, le Québec a fait des progrès immenses sur le plan de la qualité des ressources humaines. Il semble bien que le Québec devra se préoccuper au cours des prochaines décennies (il est tout à fait fortuit que les projections démographiques utilisées ici s'étendent aussi sur une période de huit décennies) non seulement de qualité mais du nombre, à tel point qu'on serait tenté de transformer la consigne d'E. Bouchette : La qualité est utile pour le présent, le nombre est essentiel pour 1 'avenir.

\section{REFERENCES BIBLIOGRAPHIQUES}

BOUCHETTE, Errol, 1906. L'indépendance économique du Canada français. Arthabaska, La Cie d'Imprimerie d'Arthabaskaville, $344 \mathrm{p}$.

BUREAU DE LA STATISTIQUE DU QUÉBEC, 1984. Perspectives démographiques régionales, 1981-2006. Québec, $436 \mathrm{p}$.

BUREAU DE LA STATISTIQUE DU QUEBEC, 1985. L'avenir démographique du Québec. Québec, 219 p.

CIURIAK, Dan et Harvey SIMS, 1980. Croissance du taux d'activité et de la population active du Canada. Ottawa, Ministère des finances du Canada, $81 \mathrm{p}$.

DENTON, Frank T., Christine H. FEAVER et Byron G. SPENCER, 1980. La population et la main-d'oeuvre au Canada. Projections 1976-2051. Ottawa, Conseil économique du Canada, $46 \mathrm{p}$.

GAUTHIER, Hervé, 1977. Evolution démographique du Québec. Québec, Office de planification et de développement du Québec, Collection "Etudes et recherches", 168 p.

GAUTHIER, Hervé et Jocelyn CôTÉ, 1980. "La population active au Québec depuis 1961 jusqu'en 1'an 2001". Cahiers québécois de démographie, $9,2,7-31$.

GAUTHIER, Hervé et Renée MALO, 1983. "Perspectives à long terme de la population québécoisell. In Démographie québécoise : passé, présent, perspectives

GOUVERNEMENT DU QUEEBEC, 1984. L'évolution de la population et ses conséquences. Québec, Secrétariat au développement social, $96 \mathrm{p}$. 
HOSKIN, Marilyn et William MISHLER, 1983. "Public Opinion toward New Migrants: A Comparativel. Migrations internationales, $\mathrm{XXI}, 4,440-462$.

KEMPSTER, A.J., 1973. Étude des données du recensement de 1971 sur la population active. Ottawa, Statistique Canada, Population et logement, Note de recherche no 71-EC,-4, 12 p.

I.E BRAS, Hervé et Georges TAPINOS, 1979. "Perspectives à long terme de la population française et leurs implications économiques". Population, 34, no spécial, 1391-1452.

McDONALD, Linda J., 1978. Changing Population and the Impact on Government Age-Specific Expenditures. Ottawa, Secrétariat du Conseil du Trésor, miméo, 89 p. et annexes.

MONNIER, Alain, 1983. "La conjoncture démographique : 1'Europe et les pays développés d'Outre-Merl. Population, 38, 4-5, $827-839$.

MONNIER, Alain, 1985. "La conjoncture démographique : 1'Europe et les pays développés d'Outre-Merl. Population, 40, 4-5, 749-763.

OFFICE DE PLANIFICATION FT DE DÉVELOPPEMENT DU QUÉBEC (OPDQ), 1980. Effets économiques du ralentissement de la croissance de la population au Québec, sous la direction de H. Gauthier, M. Frappier-Durocher et R. Durand, $187 \mathrm{p}$.

PROULX, Jean-Pierre, 1985. "La baisse de la population n'inquiète pas les Québécois". Le Devoir, 16 décembre, 1.

STATISTIQUE CANADA, 1983. Estimations de la population du Canada et des provinces, ler juin 1983. Ottawa, Approvisionnements et Services, no 91-201 au catalogue.

WANDER, Hilde, 1978. "Aspects démographiques de la population activell. In Conseil de l'Europe, Incidences d'une population stationnaire ou décroissante en Europe, Liège, Ordina, 73-101. 
RESUME - SUMMARY - RESUMEN

GAUTHIER Hervé - TROIS SCÉNARIOS DE DECROISSANCE DE LA POPULATION QUÉBÉCOISE : CARACTERISTIQUES ET INCIDENCES SUR LA POPULATION ACTIVE

Après avoir décrit différents scénarios de décroissance de la population du Québec, l'auteur fournit des exemples de déclin observés au XXe siècle, et souligne que la possibilité de recourir à l'immigration pour éviter la décroissance pourrait être limitée si la population ne modifie pas son attitude plutôt négative à l'égard d'un accroissement de l'immigration. Il analyse ensuite les implications de la décroissance sous quatre aspects : la taille de la population active, sa composition par sexe et par âge, le taux brut d'activité et le rapport de dépendance économique.

GAUTHIER Hervé - THREE PROJECTIONS OF POPULATION DECLINE FOR QUEBEC: CHARACTERISTICS AND IMPLICATIONS FOR THE WORKING POPULATION

After a brief review of various projections of population decline for Quebec, the author analyses some previous examples of depopulation, and emphasizes that public opinion will have to change much if immigration is to be used as a tool for avoiding population decline. He then investigates four implications of the projected decline: the size of the working population, its age and sex structure, the labor force participation ratio, and the economic dependency ratio.

GAUTHIER Hervé - TRES ESCENARIOS DE DECRECIMIENTO DE LA POBLACION QUEBEQUENSE : CARACTERISTICAS E INCIDENCIAS SOBRE LA POBLACION ACTIVA

Despueś de una descripción de los diferentes escenarios del decrecimiento de la población del Québec, el autor presenta algunos ejemplos de disminución observados en el siglo XX y opina que la posibilidad de recurrir a la inmigración para evitar el decrecimiento podría estar limitada si la problación no modifica su actitud de tendencia negativa hacia un crecimiento de la inmigración. E1 autor analiza tambien las implicaciones del decrecimiento bajo cuatro aspectos : 1) el tamaño de la población activa, 2) su composición por sexo y por edad, 3) la tasa de actividad y 4) la relación de dependencia económica. 